\title{
Acknowledgement to Reviewers of Fire in 2018
}

\section{Fire Editorial Office}

MDPI, St. Alban-Anlage 66, 4052 Basel, Switzerland

Published: 8 January 2019

\begin{abstract}
Rigorous peer-review is the corner-stone of high-quality academic publishing. The editorial team greatly appreciates the reviewers who contributed their knowledge and expertise to the journal's editorial process over the past 12 months. In 2018, a total of 48 papers were published in the journal, with a median time to first decision of 17 days and a median time to publication of 46 days. The editors would like to express their sincere gratitude to the following reviewers for their cooperation and dedication in 2018:
\end{abstract}

Alcasena, Fermin

Alexandre, Marty

Archibald, Sally

Aryal, Jagannath

Badik, Kevin J.

Baker, Kirk R.

Bigelow, Seth W.

Bilbao, Bibiana

Billmire, Michael

Blackhall, Melisa

Bowman, David

Bright, Ben

Butler, Corey R.

Butler, Bret W.

Campos-Ruiz, Rodrigo

Carpenter, Raymond J.

Charney, Jay

Christianson, Amy

Chu, Thuan

Cisneros, Ricardo

Cochrane, Mark A.

Conedera, Marco

Copes-Gerbitz, Kelsey

Coughlan, Michael

Cowell, Carly

Cruz, Miguel

Curran, Tim

De Almeida, Danilo Roberti Alves

Duine, Gert-Jan

Fan, Lei

Fernandes, Paulo

Filipponi, Federico

Fitch, Ryan A.

Fletcher, Thomas $\mathrm{H}$

Fovell, Robert
Fox-Hughes, Paul

Freeman, Johanna

Fréjaville, Thibaut

Garcia De Leon, David

Geary, William

Gibos, Kelsy

Gollner, Michael

Goodrick, Scott

Guo, Futao

Haire, Sandra L.

Hansen, Winslow

Harley, Grant

Hatchett, Benjamin J.

Hayasaka, Hiroshi

He, Tianhua

Heinsch, Faith Ann

Higuera, Philip

Hilton, J.E.

Hoffman, Chad

Huang, Haiyan

Hudak, Andrew T.

Iglesias, Virginia

Ingalsbee, Timothy

Iniguez, Jose $\mathrm{M}$.

Ioannidou, Effie

Jakimow, Benjamin

Jandt, Randi

Jiménez, Enrique

Johansson, Nils

Johnston, James D.

Kaplan, Jed O.

Król, Małgorzata

Lamelas, María Teresa

Lamont, Byron

Laneve, Giovanni 
Laris, Paul

LeMone, Margaret

Li, Kaiyuan

Liu, Fang

Long, Jonathan W.

Lopes Thomaz, Edivaldo

López-Serrano, Pablito M.

Lothian, Alexis

$\mathrm{Ma}$, Qin

Mallinis, Giorgios

Marx, Hannah E.

Mass, Clifford F.

McCaffrey, Sarah

McColl-Gausden, Sarah C.

McGranahan, Devan Allen

Mell, William E.

Merschel, Andrew

Miller, Eric

Mitsopoulos, Ioannis

Molina, Juan Ramón

Moreno, José M.

Mueller, Eric Victor

Nauslar, Nick

Newnham, Glenn

Nielsen-Pincus, Max

Null, Jan

Page, Wesley

Parsons, Russ

Pimont, François

Pretrel, Hugues

Price, Owen F.

Rabin, Sam

Robinne, François-Nicolas

Robinson, Guy M.
Rogers, Paul

Rolinski, Tom

Rolph, Glenn

Rossa, Carlos G.

Rother, Monica

Ruffault, J.

Särdqvist, Stefan

Sasal, Yamila

Schwilk, Dylan

Shotorban, Babak

Sopko, Paul A.

Sparks, Aaron M.

Speer, Jim

Stavros, Natasha

Tane, Zachary

Tasmin, Saira

Tedim, Fantina

Tham, Rachel

Thomas, Jan Christian

Thompson, Steve

Tinkham, Wade

Urbieta, Itziar R.

Velez, Anne-Lise K.

Walker, Anthony

Walls, Richard

Węgrzyński, Wojciech

Woody, Matthew

Wyse, Sarah V.

Xiao, Wen

Yang, Justin

Yedinak, Kara

Yousif, Osama

Yuen, Anthony Chun Yin

Zylstra, Philip

(C) 2019 by the authors. Licensee MDPI, Basel, Switzerland. This article is an open access article distributed under the terms and conditions of the Creative Commons Attribution (CC BY) license (http://creativecommons.org/licenses/by/4.0/). 